\title{
A Characterization of Orthonormal Multilevel Wavelet Families in Sobolev Space over Local Fields of Positive Characteristic
}

\author{
Ashish Pathak and Dileep Kumar
}

\begin{abstract}
In this paper, a characterization of orthonormal multilevel wavelet families in Sobolev space over a local fields of positive characteristic $\left(H^{s}(\mathbb{K})\right)$ is established. Finally an example is presented.
\end{abstract}

\section{Introduction}

The idea of wavelets as a family of function constructed by using translation and dilation of a single function was given by Morlet in 1982. The wavelet on $L^{2}(\mathbb{R})$ were characterized by Wang [27] and Gripenberg [17] with the help of Fourier transform of the wavelet. Bownik [21] obtained new concept to characterizing multiwavelets in $L^{2}\left(\mathbb{R}^{n}\right)$ by using the results of shift invariant systems and quasi-affine systems in ([8], [9], [14], [22] ).

Jiang, Li and Jin discussed multiresolution analysis on local fields of positive characteristic and constructed corresponding orthonormal wavelets (see[18]).Behera and Jahan were first generalized the concept of multiresolution analysis (MRA) and wavelets in the space $L^{2}(\mathbb{K})$ and established orthonormal basis from Riesz basis (see [11]).They were also characterized the wavelets and MRA wavelets over a local fields of positive characteristic by affine frame systems and quasiaffine systems (see [12]). Pathak and Singh recently modified the classical definition of multiresolution analysis and construct the orthonormal wavelet in Sobolev space over local fields of positive characteristic $\left(H^{s}(\mathbb{K})\right)$ in [5].

In this paper, we characterized the orthonormal multilevel wavelet families in Sobolev space over a local fields of positive characteristic. This article is organized as follows. In section 2, we give some basic notations and definitions of local field and Sobolev space over local fields. In section 3, this section contains the basic definition of orthonormal wavelet in $H^{s}(\mathbb{K})$ and four lemmas, by using these lemmas we will prove the main result of this paper. Finally in this section we characterized the orthonormal multilevel wavelet families in Sobolev space over a local fields of positive characteristic $\left(H^{s}(\mathbb{K})\right)$ and an example is presented.

Key words and phrases. Wavelets ; Orthonormal wavelet families; Local fields; Sobolev space; Fourier transform;.

2010 Mathematics Subject Classification. 42C40; 46E35; $11 \mathrm{~F} 87$.

Corresponding author: Ashish Pathak. 


\section{Notation and definitions}

A local field $\mathbb{K}$ means an algebraic field and a topological space with the properties of complete, locally compact, totally disconnected and non-discrete and Haar measure on $\mathbb{K}^{+}$is denoted by $d x$. The absolute value or valuation of $x$ is denoted by $|x|$ and have the properties (i) $|x| \geq 0$ and $|x|=0$ iff $x=0$;(ii) $|x y|=|x||y|$; (iii) $|x+y| \leq \max (|x|,|y|)$.

Now, we recall some Notations which are used in this paper

- Throughout this paper $\mathbb{K}$ denotes the local field of positive characteristic.

- $d x$ is the normalized Haar measure for $\mathbb{K}^{+}$.

- $|\alpha|$ is the valuation of $\alpha \in \mathbb{K}$ and it is non-archimedian norm.

- We will use following notations for the numbers, $\mathbb{Z}=$ the set of integers; $\mathbb{N}=$ the set of natural numbers; $\mathbb{N}_{0}=\{0,1,2,3, \ldots\}$.

- Let $\mathfrak{s}$ be a prime element in $\mathbb{K}$.

- For $k \in \mathbb{Z}, \mathfrak{P}^{k}=\left\{x \in \mathbb{K}:|x| \leq q^{-k}\right\}$ is a compact subgroup of $\mathbb{K}^{+} \cdot \mathfrak{P}^{0}=\mathfrak{D}$ is called ring of integres in $\mathbb{K}$.

- $\left|\mathfrak{P}^{k}\right|=q^{-k}$ and $|\mathfrak{D}|=1$.

- $\chi$ be a fixed character on $\mathbb{K}^{+}$that is trivial on $\mathfrak{D}$ but is non trivial on $\mathfrak{P}^{-1}$. For $y \in \mathbb{K}$, $\chi_{y}(x)=\chi(y x), x \in \mathbb{K}$.

- The "natural"order on the sequence is denoted by $\{\omega(k) \in \mathbb{K}\}_{k=0}^{\infty}$ and is described as follows.

$\mathfrak{D} / \mathfrak{P} \cong G F(q)=\tau, q=p^{s}, p$ is a prime, $s \in \mathbb{N}$ and $\Omega: \mathfrak{D} \rightarrow \tau$ the canonical homomorphism of $\mathfrak{D}$ on to $\tau$. $\tau=G F(q)$ is a vector space over $G F(p) \subset \tau$. We consider a set $\left\{1=\epsilon_{0}, \epsilon_{1}, \ldots, \epsilon_{s-1}\right\} \subset \mathfrak{D}^{*}=\mathfrak{D} \backslash \mathfrak{P}$ in such a way that $\left\{\Omega\left(\epsilon_{k}\right)\right\}_{k=0}^{s-1}$ is a basis of $G F(q)$ over $G F(p)$.

For $k, 0 \leq k<q, k=a_{0}+a_{1} p+\ldots+a_{s-1} p^{s-1}, 0 \leq a_{i}<p, i=0,1, \ldots, s-1$, we define

$$
\omega(k)=\left(a_{0}+a_{1} \epsilon_{1}+\ldots+a_{s-1} \epsilon_{c-1}\right) \mathfrak{s}^{-1}(0 \leq k<q) .
$$

For $k=b_{0}+b_{1} q+\ldots+b_{r} q^{r}, 0 \leq b_{i}<q, k \geq 0$, we set

$$
\omega(k)=\omega\left(b_{0}\right)+\mathfrak{s}^{-1} \omega\left(b_{1}\right)+\ldots+\mathfrak{s}^{-r} \omega\left(b_{r}\right) .
$$

Remark 1. A function $g$ defined on $\mathbb{K}$ is integral-periodic if $g(x+\omega(n))=g(x)$ for all $n \in \mathbb{N}_{0}$.

Proposition 2.1. For all $l, k \in \mathbb{N}_{0}, \chi_{k}(\omega(l))=1$. 


\subsection{Distributions over local fields}

The space $\mathscr{S}^{\prime}(\mathbb{K})$ of continuous linear functional on $\mathscr{S}(\mathbb{K})$ (the space of all finite linear combinations of characteristics functions of ball of $\mathbb{K}$ ) is called the space of distributions. The Fourier transform of $f \in \mathscr{S}(\mathbb{K})$ is denoted by $\hat{f}(\xi)$ and defined by the

$$
\hat{f}(\xi)=\int_{\mathbb{K}} f(x) \overline{\chi_{\xi}(x)} d x=\int_{\mathbb{K}} f(x) \chi(-\xi x) d x, \xi \in \mathbb{K},
$$

and the inverse Fourier transform by

$$
f(x)=\int_{\mathbb{K}} \hat{f}(\xi) \chi_{x}(\xi) d \xi, x \in \mathbb{K} .
$$

The Fourier transform and inverse Fourier transforms of a distributions $f \in \mathscr{S}^{\prime}(\mathbb{K})$ is defined by

$$
\langle\hat{f}, \phi\rangle=\langle f, \hat{\phi}\rangle,\left\langle f^{\vee}, \phi\right\rangle=\left\langle f, \phi^{\vee}\right\rangle \text {, for all } \phi \in \mathscr{S}(\mathbb{K}) \text {. }
$$

Definition 1. Sobolev space over local fields $\left(H^{s}(\mathbb{K})\right)$.

Let $s \in \mathbb{R}$, we denote by $H^{s}(\mathbb{K})$ is the space of all $f \in \mathscr{S}^{\prime}(\mathbb{K})$ such that

$$
\hat{\nu}^{\frac{s}{2}}(\xi) \hat{f}(\xi) \in L^{2}(\mathbb{K}) .
$$

Obviously, for any real number $s, H^{s}(\mathbb{K})$ is a linear space. We equip $H^{s}(\mathbb{K})$ with the inner product

$$
\langle f, g\rangle_{s}=\langle f, g\rangle_{H^{s}(\mathbb{K})}=\int_{\mathbb{K}} \hat{\nu}^{s}(\xi) \hat{f}(\xi) \overline{\hat{g}(\xi)} d \xi,
$$

which induces the norm

$$
\|f\|_{H^{s}(\mathbb{K})}^{2}=\int_{\mathbb{K}} \hat{\nu}^{s}(\xi)|\hat{f}(\xi)|^{2} d \xi .
$$

Theorem 2.1. The space $\mathscr{S}(\mathbb{K})$ is dense in $H^{s}(\mathbb{K})$.

Proof. See [5].

\section{Main Result}

The aim of this section to established the condition which enable us to characterize all orthonormal wavelet families in $H^{s}(\mathbb{K})$. Before characterization of orthonormal wavelet, we first define the orthonormal wavelets and recall four lemmas which will we used in the proof of main result. Definition 2. Let $\left\{\psi_{r}^{(j)}\right\}_{j \in \mathbb{Z}, r \in D_{1}} \subseteq H^{s}(\mathbb{K})$. Then $\left\{\psi_{r}^{(j)}\right\}_{j \in \mathbb{Z}, r \in D_{1}}$ is said to be an orthonormal wavelet family in $H^{s}(\mathbb{K})$ if $H^{s}(\mathbb{K})=\overline{\operatorname{span}\left\{\psi_{r, j, k}^{(j)}\right\}_{r \in D_{1}, j \in \mathbb{Z}, k \in \mathbb{N}_{0}}} \quad$ where $D_{1}=\{1,2,3, \ldots q-1\}$ and $\left\langle\psi_{r, j, k}^{(j)}, \psi_{r, l, m}^{(l)}\right\rangle=\delta_{j, l} \delta_{k, m} \quad$ for all $j, l \in \mathbb{Z}$ and $k, m \in \mathbb{N}_{0}$, where both $\delta_{j, l}$ and $\delta_{k, m}$ are the Kronecker delta functions. 
Lemma 3.1. Let a function $f$ belonging to dense subset $\mathscr{S}(\mathbb{K})$ of Sobolev space $H^{s}(\mathbb{K})$ and a set $\left\{v_{j}: j=1,2,3, \ldots\right\}$ is the system of vectors in $H^{s}(\mathbb{K})$ such that

$$
\|f\|_{H^{s}(\mathbb{K})}^{2}=\sum_{j=1}^{\infty}\left|\left\langle f, v_{j}\right\rangle\right|^{2}
$$

then equality (3.1) holds for every $f \in H^{s}(\mathbb{K})$ (see [16]).

Lemma 3.2. Let a set $\left\{v_{j}: j=1,2,3, \ldots\right\}$ is the system of vectors in $H^{s}(\mathbb{K})$ satisfying equality (3.1). If $\left\|v_{j}\right\| \geq 1$ for $i=1,2,3, \ldots$, then $\left\{v_{j}: j=1,2,3, \ldots\right\}$ is an orthonormal basis for $H^{s}(\mathbb{K})$ (see [16]).

Lemma 3.3. If $f \in \mathscr{S}(\mathbb{K})$ and $\psi_{r}^{(j)} \in H^{s}(\mathbb{K})$, then

$$
\begin{aligned}
\sum_{r \in D_{1}} \sum_{k \in \mathbb{N}_{0}}\left|\left\langle f, \psi_{r, j, k}^{(j)}\right\rangle_{H^{s}(\mathbb{K})}\right|^{2}=\sum_{r \in D_{1}} \int_{\mathbb{K}} \hat{\nu}^{s}(\xi) \overline{\hat{f}(\xi)} \hat{\psi}_{r}^{(j)}\left(\mathfrak{s}^{-j} \xi\right)\left\{\sum_{k \in \mathbb{N}_{0}} \hat{\nu}^{s}\left(\xi+\mathfrak{s}^{-j} \omega(k)\right)\right. \\
\left.\times \hat{f}\left(\xi+\mathfrak{s}^{-j} \omega(k)\right) \overline{\hat{\psi}_{r}^{(j)}\left(\mathfrak{s}^{j} \xi+\omega(k)\right)}\right\} d \xi
\end{aligned}
$$

Proof. See [4].

Lemma 3.4. Let $f$ be in $\mathscr{S}(\mathbb{K})$ and $\psi_{r}^{(j)} \in H^{s}(\mathbb{K})$. If ess $\sup \left\{\hat{\nu}^{s}(\xi) \sum_{r \in D_{1}} \sum_{j \in \mathbb{Z}}\left|\hat{\psi}_{r}^{(j)}\left(\mathfrak{s}^{j} \xi\right)\right|^{2}\right.$ : $\left.\xi \in \mathfrak{P}^{-1} \backslash \mathfrak{D}\right\}<\infty$, then

$$
\sum_{r \in D_{1}} \sum_{j \in \mathbb{Z}} \sum_{k \in \mathbb{N}_{0}}\left|\left\langle f, \psi_{r, j, k}^{(j)}\right\rangle\right|^{2}=\int_{\mathbb{K}} \hat{\nu}^{2 s}(\xi)|\hat{f}(\xi)|^{2} \sum_{r \in D_{1}} \sum_{j \in \mathbb{Z}}\left|\hat{\psi}_{r}^{(j)}\left(\mathfrak{s}^{j} \xi\right)\right|^{2} d \xi+T_{2},
$$

where

$$
\begin{array}{r}
T_{2}=\sum_{r \in D_{1}} \sum_{j \in \mathbb{Z}} \int_{\mathbb{K}} \hat{\nu}^{s}(\xi) \overline{\hat{f}(\xi)} \hat{\psi}_{r}^{(j)}\left(\mathfrak{s}^{j} \xi\right)\left[\sum_{l \in \mathbb{N}} \hat{\nu}^{s}\left(\xi+\mathfrak{s}^{-j} \omega(l)\right) \hat{f}\left(\xi+\mathfrak{s}^{-j} \omega(l)\right)\right. \\
\left.\times \overline{\hat{\psi}_{r}^{(j)}\left(\mathfrak{s}^{j} \xi+\omega(l)\right)}\right] d \xi .
\end{array}
$$

Furthermore, the iterated series in (3.4) is absolutely convergent.

Proof. See [4].

Theorem 3.1. Let $\left\{\psi_{r}^{(j)}\right\}_{j \in \mathbb{Z}, r \in D_{1}} \subseteq H^{s}(\mathbb{K})$, with $\left\|\psi_{r}^{(j)}\right\|_{H^{s}(\mathbb{K})}=1, j \in \mathbb{Z}, r \in D_{1}$. Then the collection $\left\{\psi_{r}^{(j)}\right\}_{j \in \mathbb{Z}, r \in D_{1}}$ is an orthonormal wavelet families in $H^{s}(\mathbb{K})$ if and only if

$$
\sum_{r \in D_{1}} \sum_{j \in \mathbb{Z}}\left|\hat{\psi}_{r}^{(j)}\left(\mathfrak{s}^{j} \xi\right)\right|=\nu^{-s}(\xi) \text { for a.e. } \xi \in \mathbb{K},
$$

and for every $s \in \mathbb{N}_{0} \backslash q \mathbb{N}_{0}$,

$$
\sum_{r \in D_{1}} \sum_{i=0}^{\infty} \overline{\hat{\psi}_{r}^{(j-i)}\left(\mathfrak{s}^{j-i} \xi\right)} \hat{\psi}_{r}^{(j-i)}\left(\mathfrak{s}^{-i}\left(\mathfrak{s}^{j} \xi+\omega(s)\right)\right)=0
$$

for a.e. $\xi \in \mathbb{K}$ and for all $s \in \mathbb{N}_{0} \backslash q \mathbb{N}_{0}$, where $q \mathbb{N}_{0}=\left\{q k: k \in \mathbb{N}_{0}\right\}$. 
Proof. Suppose that equalities (3.5) and (3.6) holds. Then by Lemma 3.1 and 3.2, it is sufficient to show that

$$
\sum_{r \in D_{1}} \sum_{k \in \mathbb{N}_{0}} \sum_{j \in \mathbb{Z}}\left|\left\langle f, \psi_{r, j, k}^{(j)}\right\rangle_{H^{s}(\mathbb{K})}\right|^{2}=\|f\|_{H^{s}(\mathbb{K})}^{2}
$$

hold for each $f$ belonging to $\mathscr{S}(\mathbb{K})$.

We have

$$
\begin{aligned}
& T=\sum_{r \in D_{1}} \sum_{k \in \mathbb{N}_{0}} \sum_{j \in \mathbb{Z}}\left|\left\langle f, \psi_{r, j, k}^{(j)}\right\rangle_{H^{s}(\mathbb{K})}\right|^{2} \\
& =\sum_{r \in D_{1}} \sum_{k \in \mathbb{N}_{0}} \sum_{j \in \mathbb{Z}}\left|\int_{\mathbb{K}} \hat{\nu}^{s}(\xi) \hat{f}(\xi) q^{\frac{j}{2}} \overline{\hat{\psi}_{r}^{(j)}\left(\mathfrak{s}^{-j} \xi-\omega(k)\right)} d \xi\right|^{2} \\
& =\sum_{r \in D_{1}, k \in \mathbb{N}_{0}, j \in \mathbb{Z}}\left|\int_{\mathbb{K}} \hat{\nu}^{s}\left(\mathfrak{s}^{-j} \xi\right) \hat{f}\left(\mathfrak{s}^{-j} \xi\right) q^{\frac{j}{2}} \hat{\psi}_{r}^{(j)}(\xi) \chi_{k}(\xi) d \xi\right|^{2} \\
& =\sum_{r \in D_{1}, k \in \mathbb{N}_{0}, j \in \mathbb{Z}} q^{j} \int_{\mathbb{K}}\left\{\sum_{l=0}^{\infty} \int_{\mathfrak{D}} \hat{\nu}^{s}\left(\mathfrak{s}^{-j}(\xi+\omega(l))\right) \hat{f}\left(\mathfrak{s}^{-j}(\xi+\omega(l))\right) \chi_{k}(\xi+\omega(l))\right. \\
& \left.\times \overline{\hat{\psi}_{r}^{(j)}(\xi+\omega(l))} d \xi\right\} \hat{\nu}^{s}\left(\mathfrak{s}^{-j} \xi\right) \overline{\hat{f}\left(\mathfrak{s}^{-j} \xi\right)} \hat{\psi}_{r}^{(j)}(\xi) \bar{\chi}_{k}(\xi) d \xi .
\end{aligned}
$$

Since $f \in \mathscr{S}(\mathbb{K})$ so that $\sum_{l=0}^{\infty}$ contains only finite non-zero terms and $\chi_{k}(\omega(l))=1$ for $k, l \in \mathbb{N}_{0}$ , then we get

$$
\begin{array}{r}
T=\sum_{r \in D_{1}, k \in \mathbb{N}_{0}, j \in \mathbb{Z}} q^{j} \int_{\mathbb{K}}\left\{\int_{\mathfrak{D}}\left(\sum_{l=0}^{\infty} \hat{\nu}^{s}\left(\mathfrak{s}^{-j}(\xi+\omega(l))\right) \hat{f}\left(\mathfrak{s}^{-j}(\xi+\omega(l))\right) \overline{\hat{\psi}_{r}^{(j)}(\xi+\omega(l))}\right)\right. \\
\left.\times \chi_{k}(\xi) d \xi\right\} \hat{\nu}^{s}\left(\mathfrak{s}^{-j} \xi\right) \overline{\hat{f}\left(\mathfrak{s}^{-j} \xi\right)} \hat{\psi}_{r}^{(j)}(\xi) \bar{\chi}_{k}(\xi) d \xi .
\end{array}
$$

By convergence theorem of Fourier series on $\mathfrak{D}$, we obtain

$$
\begin{aligned}
T & =\sum_{r \in D_{1}, j \in \mathbb{Z}} \int_{\mathbb{K}} \hat{\nu}^{s}(\xi) \overline{\hat{f}(\xi)} \hat{\psi}_{r}^{(j)}(\xi)\left\{\sum_{l=0}^{\infty} \hat{\nu}^{s}\left(\xi+\mathfrak{s}^{-j} \omega(l)\right) \hat{f}\left(\xi+\mathfrak{s}^{-j} \omega(l)\right) \overline{\hat{\psi}_{r}^{(j)}\left(\mathfrak{s}^{j} \xi+\omega(l)\right)}\right\} d \xi \\
= & \sum_{r \in D_{1}, j \in \mathbb{Z}} \int_{\mathbb{K}} \hat{\nu}^{2 s}(\xi)|\hat{f}(\xi)|^{2}\left|\hat{\psi}_{r}^{(j)}(\xi)\right|^{2} d \xi+\int_{\mathbb{K}} \sum_{r \in D_{1}, j \in \mathbb{Z}} \sum_{l=1}^{\infty} \hat{\nu}^{s}(\xi) \hat{\nu}^{s}\left(\xi+\mathfrak{s}^{-j} \omega(l)\right) \\
= & \quad \times \hat{f}(\xi) \overline{\hat{\psi}_{r}^{(j)}\left(\mathfrak{s}^{j} \xi\right)} \overline{\hat{f}\left(\xi+\mathfrak{s}^{-j} \omega(l)\right)} \hat{\psi}_{r}^{(j)}\left(\mathfrak{s}^{j} \xi+\omega(l)\right) d \xi \\
&
\end{aligned}
$$

where

$$
T_{1}=\int_{\mathbb{K}} \hat{\nu}^{2 s}(\xi)|\hat{f}(\xi)|^{2} \sum_{r \in D_{1}} \sum_{j \in \mathbb{Z}}\left|\hat{\psi}_{r}^{(j)}\left(\mathfrak{s}^{j} \xi\right)\right|^{2} d \xi
$$


and

$$
T_{2}=\sum_{r \in D_{1}} \sum_{j \in \mathbb{Z}} \int_{\mathbb{K}} \hat{\nu}^{s}(\xi) \hat{f}(\xi) \overline{\hat{\psi}_{r}^{(j)}\left(\mathfrak{s}^{j} \xi\right)} \sum_{l=1}^{\infty} \hat{\nu}^{s}\left(\xi+\mathfrak{s}^{-j} \omega(l)\right) \overline{\hat{f}\left(\xi+\mathfrak{s}^{-j} \omega(l)\right)} \hat{\psi}_{r}^{(j)}\left(\mathfrak{s}^{j} \xi+\omega(l)\right) d \xi .
$$

Now by equality (3.5) and (3.10), we get

$$
T_{1}=\int_{\mathbb{K}} \hat{\nu}^{s}(\xi)|\hat{f}(\xi)|^{2} d \xi=\|f\|_{H^{s}(\mathbb{K})}^{2} .
$$

Now we need only to show that $T_{2}=0$. Since $T_{2}$ is absolutely convergent. In the expression of $T_{2}, l \in \mathbb{N}$,for given $l$ there exist $i \in \mathbb{N}_{0}$ and a unique $m \in q \mathbb{N}_{0}+\tilde{Q}$ such that $l=q^{i} m$ where $\tilde{Q}=\{1,2,3, \ldots q-1\}$. Thus by Definition 2, we have $\{\omega(l)\}_{l \in \mathbb{N}}=\left\{\mathfrak{s}^{i} \omega(m)\right\}_{(i, m) \in \mathbb{N}_{0} \times\left\{q \mathbb{N}_{0}+\tilde{Q}\right\}}$. Since the series in (3.11) is absolutely convergent. Therefore equation (3.11) become

$$
\begin{gathered}
T_{2}=\sum_{r \in D_{1}} \sum_{j \in \mathbb{Z}} \int_{\mathbb{K}} \hat{\nu}^{s}(\xi) \hat{f}(\xi) \overline{\hat{\psi}_{r}^{(j)}\left(\mathfrak{s}^{j} \xi\right)}\left\{\sum_{i \in \mathbb{N}_{0}} \sum_{m \in q \mathbb{N}_{0}+\tilde{Q}} \hat{\nu}^{s}\left(\xi+\mathfrak{s}^{-j-i} \omega(m)\right) \overline{\hat{f}\left(\xi+\mathfrak{s}^{-j-i} \omega(m)\right)}\right. \\
=\int_{\mathbb{K}} \hat{\nu}^{s}(\xi) \hat{f}(\xi)\left\{\sum_{j \in \mathbb{Z}} \sum_{m \in q \mathbb{N}_{0}+\tilde{Q}} \hat{\nu}_{r}^{s}\left(\xi+\mathfrak{s}^{j} \xi+\mathfrak{s}^{-i} \omega(m)\right)\right\} d \xi \\
\left.\times \sum_{r \in D_{1}} \sum_{i \in \mathbb{N}_{0}} \overline{\hat{\psi}_{r}^{(j-i)}\left(\mathfrak{s}^{j-i} \xi\right)} \hat{\psi}_{r}^{(j-i)}\left(\mathfrak{s}^{j-i} \xi+\mathfrak{s}^{-i} \omega(m)\right)\right\} d \xi .
\end{gathered}
$$

Now using equation (3.6), we get $T_{2}=0$.

Conversely, suppose that $\left\{\psi_{r}^{(j)}: j \in \mathbb{Z}, r \in D_{1}\right\}$ is the orthonormal wavelet family in $H^{s}(\mathbb{K})$. Then we show that both equations (3.5) and (3.6) are satisfied. Since $\left\{\hat{\psi}_{r}^{(j)}\right\}_{j \in \mathbb{Z}, r \in D_{1}}$ is orthonormal wavelet. Therefore for all $f \in \mathscr{S}(\mathbb{K})$, we have

$$
\sum_{r \in D_{1}} \sum_{k \in \mathbb{N}_{0}} \sum_{j \in \mathbb{Z}}\left|\left\langle f, \psi_{r, j, k}^{(j)}\right\rangle_{H^{s}(\mathbb{K})}\right|^{2}=\|f\|_{H^{s}(\mathbb{K})}^{2}=T_{1}+T_{2}
$$

Using the fact that $\left|T_{2}\right|<\infty$, we have

$$
T_{1}=\sum_{r \in D_{1}} \sum_{j \in \mathbb{Z}} \int_{\mathbb{K}} \hat{\nu}^{2 s}(\xi)|\hat{f}(\xi)|^{2}\left|\hat{\psi}_{r}^{(j)}\left(\mathfrak{s}^{j} \xi\right)\right|^{2} d \xi<\infty
$$

By the assumption of $f \in \mathscr{S}(\mathbb{K})$,we can obtain that $\hat{\nu}^{s}(\xi)|\hat{f}(\xi)|$ is a bounded function. So $\hat{\nu}^{s}(\xi) \sum_{r \in D_{1}} \sum_{j \in \mathbb{Z}}\left|\hat{\psi}_{r}^{(j)}\left(\mathfrak{s}^{j} \xi\right)\right|^{2}$ is localy integrable in $\mathbb{K} \backslash \bigcup_{j \in \mathbb{Z}} E_{j}^{c}$, where $E_{j}$ is the set of regular points of $\left|\psi_{r}^{(j)}\left(\mathfrak{s}^{j} \xi\right)\right|$ (see[23], pp. 29). Therefore each $\xi_{0} \in \mathbb{K} \backslash \bigcup_{j \in \mathbb{Z}} E_{j}^{c}$, we consider

$$
\hat{g}(\xi)=q^{\frac{M}{2}} Q_{m}\left(\xi-\xi_{0}\right) \hat{\nu}^{\frac{-s}{2}}(\xi)
$$


where $f=g$ and $Q_{m}\left(\xi-\xi_{0}\right)$ is the characteristic function of $\xi_{0}+\mathfrak{P}^{M}$. Then it follows that $\overline{\hat{f}}(\xi) \hat{f}\left(\xi+\mathfrak{s}^{-j} \omega(l)\right)=0$ for $l \in \mathbb{N}$. Since $\xi$ and $\xi+\mathfrak{s}^{-j} \omega(l)$ can not be in $\xi_{0}+\mathfrak{P}^{M}$ simultaneously, and hence $\|g\|_{H^{s}(\mathbb{K})}=1$. Further more, we have

$$
1=\|g\|_{H^{s}(\mathbb{K})}^{2}=\int_{\xi_{0}+\mathfrak{P}^{M}} \hat{\nu}^{s}(\xi) \sum_{r \in D_{1}} \sum_{j \in \mathbb{Z}} q^{M}\left|\hat{\psi}_{r}^{(j)}\left(\mathfrak{s}^{j} \xi\right)\right|^{2} d \xi+T_{2}
$$

and let $M \rightarrow \infty$, we obtain

$$
1=\hat{\nu}^{s}\left(\xi_{0}\right) \sum_{r \in D_{1}} \sum_{j \in \mathbb{Z}}\left|\hat{\psi}_{r}^{(j)}\left(\mathfrak{s}^{j} \xi_{0}\right)\right|^{2}+\lim _{M \rightarrow \infty} T_{2} .
$$

Now we need to show that $T_{2} \rightarrow 0$ as $M \rightarrow \infty$.Now, we estimate $T_{2}$ as follows

$$
\begin{aligned}
\left|T_{2}\right| \leq \sum_{r \in D_{1}} \sum_{j \in \mathbb{Z}} \int_{\mathbb{K}} \hat{\nu}^{s}(\xi)|\hat{f}(\xi)|\left|\hat{\psi}_{r}^{(j)}\left(\mathfrak{s}^{j} \xi\right)\right|\left\{\sum_{l=1}^{\infty} \hat{\nu}^{s}\left(\xi+\mathfrak{s}^{-j} \omega(l)\right)\left|\hat{f}\left(\xi+\mathfrak{s}^{-j} \omega(l)\right)\right|\right. \\
\left.\times\left|\hat{\psi}_{r}^{(j)}\left(\mathfrak{s}^{j} \xi+\omega(l)\right)\right|\right\} d \xi \\
=\sum_{r \in D_{1}} \sum_{j \in \mathbb{Z}} \sum_{l=1}^{\infty} q^{j} \int_{\mathbb{K}} \hat{\nu}^{s}\left(\mathfrak{s}^{-j} \xi\right)\left|\hat{f}\left(\mathfrak{s}^{-j} \xi\right)\right|\left|\hat{\psi}_{r}^{(j)}(\xi)\right| \hat{\nu}^{s}\left(\mathfrak{s}^{-j}(\xi+\omega(l))\right)\left|\hat{f}\left(\mathfrak{s}^{-j}(\xi+\omega(l))\right)\right| \\
\times \sum_{r \in D_{1}} \sum_{j \in \mathbb{Z}} \sum_{l=1}^{\infty} q^{j} \int_{\mathbb{K}} \hat{\nu}^{\frac{s}{2}}\left(\mathfrak{s}^{-j} \xi\right)|\hat{f}(\xi+\omega(l))| d \xi \\
\left.\quad \times \hat{\mathfrak{\nu}}^{-j} \xi\right)\left.\left|\hat{\nu}^{\frac{s}{2}}\left(\mathfrak{s}^{-j} \xi\right)\right| \hat{\psi}_{r}^{(j)}(\xi)\right|^{2} d \xi .
\end{aligned}
$$

Since $\omega(l) \neq 0(l \in \mathbb{N})$ and $f \in \mathscr{S}(\mathbb{K})$, there exist a constant $J>0$ such that $\hat{f}\left(\mathfrak{s}^{-j} \xi\right) \hat{f}\left(\mathfrak{s}^{-j} \xi+\mathfrak{s}^{-j} \omega(l)\right)=0$ for $|j|>J$.

Therefore series in equation (3.18) have only a finite number of terms are nonzero so there exist a constant $K$ such that

$$
\begin{aligned}
\left|T_{2}\right| & \leq K\left\|\hat{\nu}^{s}(\xi) \hat{f}(\xi)\right\|_{\infty}^{2}\left\|\hat{\psi}_{r}^{(j)}(\xi)\right\|_{H^{s}(\mathbb{K})}^{2} \\
& =K q^{m}\left\|\hat{\psi}_{r}^{(j)}(\xi)\right\|_{H^{s}(\mathbb{K})}^{2}
\end{aligned}
$$

and implies that $T \rightarrow 0$ as $M \rightarrow \infty$. Hence equation (3.17) becomes

$$
\sum_{r \in D_{1}} \sum_{j \in \mathbb{Z}}\left|\hat{\psi}_{r}^{(j)}\left(\mathfrak{s}^{j} \xi_{0}\right)\right|=\hat{\nu}^{-s}\left(\xi_{0}\right)
$$

Now we show that if

$$
\sum_{r \in D_{1}} \sum_{j \in \mathbb{Z}} \sum_{k \in \mathbb{N}_{0}}\left|\left\langle f, \psi_{r, j, k}^{(j)}\right\rangle_{H^{s}(\mathbb{K})}\right|^{2}=\|f\|_{H^{s}(\mathbb{K})}^{2}
$$


holds for all $f \in \mathscr{S}(\mathbb{K})$, then the equation (3.6) holds. Now by using (3.7),(3.9) and (3.11) we have

$$
\begin{aligned}
& \sum_{j \in \mathbb{Z}} \sum_{m \in q \mathbb{N}_{0}+\tilde{Q}} \int_{\mathbb{K}} \hat{\nu}^{s}(\xi) \hat{f}(\xi) \hat{\nu}^{s}\left(\xi+\mathfrak{s}^{-j} \omega(m)\right) \overline{\hat{f}\left(\xi+\mathfrak{s}^{-j} \omega(m)\right)} \\
& \times\left\{\sum_{r \in D_{1}} \sum_{i \in \mathbb{N}_{0}} \overline{\hat{\psi}_{r}^{(j-i)}}\left(\mathfrak{s}^{j-i} \xi\right) \hat{\psi}_{r}^{(j-i)}\left(\mathfrak{s}^{-i}\left(\mathfrak{s}^{j} \xi+\omega(m)\right)\right)\right\} d \xi=0 .
\end{aligned}
$$

By Polarisation, we have

$$
\begin{aligned}
& \sum_{j \in \mathbb{Z}} \sum_{m \in q \mathbb{N}_{0}+\tilde{Q}} \int_{\mathbb{K}} \hat{\nu}^{s}(\xi) \hat{f}(\xi) \hat{\nu}^{s}\left(\xi+\mathfrak{s}^{-j} \omega(m)\right) \overline{\hat{g}\left(\xi+\mathfrak{s}^{-j} \omega(m)\right)} \\
& \times\left\{\sum_{r \in D_{1}} \sum_{i \in \mathbb{N}_{0}}{\overline{\hat{\psi}_{r}^{(j-i)}}}^{\left.\left(\mathfrak{s}^{j-i} \xi\right) \hat{\psi}_{r}^{(j-i)}\left(\mathfrak{s}^{-i}\left(\mathfrak{s}^{j} \xi+\omega(m)\right)\right)\right\} d \xi=0 .}\right.
\end{aligned}
$$

Let us fix $k_{0} \in q \mathbb{N}_{0}+\tilde{Q}$ and $\xi_{0} \in \mathbb{K} \backslash \bigcup_{j \in \mathbb{Z}} E_{j}^{c}$ such that neither $\xi_{0} \neq 0$ nor $\xi_{0}+\omega\left(k_{0}\right) \neq 0$. Consider $\hat{f}(\xi)=q^{\frac{M}{2}} \phi\left(\xi-\xi_{0}\right) \hat{\nu}^{\frac{-s}{2}}(\xi)$ and $\hat{g}(\xi)=\hat{f}\left(\xi-\omega\left(k_{0}\right)\right) \hat{\nu} \frac{-s}{2}\left(\xi-\omega\left(k_{0}\right)\right)$. We obtain $\hat{f}(\xi) \hat{g}\left(\xi+\omega\left(k_{0}\right)\right)=q^{\frac{M}{2}} \phi_{M}\left(\xi-\xi_{0}\right) \hat{\nu}^{-s}(\xi) \hat{\nu}^{-s}\left(\xi-\omega\left(k_{0}\right)\right)$.

Now, equation (3.22) can be written as

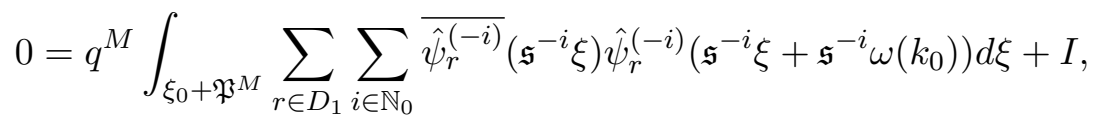

where

$$
\begin{aligned}
I= & \sum_{\substack{j \in \mathbb{Z} \\
(j, m) \neq\left(0, k_{0}\right)}} \sum_{m \in q \mathbb{N}_{0}+\tilde{Q}} \int_{\mathbb{K}} \hat{\nu}^{s}(\xi) \hat{f}(\xi) \hat{\nu}^{s}\left(\xi+\mathfrak{s}^{-j} \omega(m)\right) \overline{\hat{g}\left(\xi+\mathfrak{s}^{-j} \omega(m)\right)} \\
& \times\left\{\sum_{r \in D_{1}} \sum_{i \in \mathbb{N}_{0}} \overline{\hat{\psi}_{r}^{(j-i)}}\left(\mathfrak{s}^{j-i} \xi\right) \times \hat{\psi}_{r}^{(j-i)}\left(\mathfrak{s}^{j-i} \xi+\mathfrak{s}^{-i} \omega(m)\right)\right\} d \xi .
\end{aligned}
$$

Since the first term in (3.23) is zero.So we only to show that $I \rightarrow 0$ as $M \rightarrow \infty$. Since $\omega(m) \neq$ $0(m \in \mathbb{N})$ and $f, g \in \mathscr{S}(\mathbb{K})$, there exist a constant $J_{0}>0$ such that

$$
\hat{f}(\xi) \overline{\hat{g}(\xi+\omega(m))}=0 \forall j>J_{0} .
$$

Therefore we have

$$
\begin{aligned}
I= & \sum_{j \leq J_{0}} \sum_{m \in q \mathbb{N}_{0}+\tilde{Q}} \int_{\mathbb{K}} \hat{\nu}^{s}(\xi) \hat{f}(\xi) \hat{\nu}^{s}\left(\xi+\mathfrak{s}^{-j} \omega(m)\right) \overline{\hat{g}\left(\xi+\mathfrak{s}^{-j} \omega(m)\right)}\left\{\sum_{r \in D_{1}} \sum_{i \in \mathbb{N}_{0}} \overline{\hat{\psi}_{r}^{(j-i)}}\left(\mathfrak{s}^{j-i} \xi\right)\right. \\
& \left.\times \hat{\psi}_{r}^{(j-i)}\left(\mathfrak{s}^{j-i} \xi+\mathfrak{s}^{-i} \omega(m)\right)\right\} d \xi, \\
|I| \leq & \sum_{j \leq J_{0}} \sum_{m \in q \mathbb{N}_{0}+\tilde{Q}} q^{j} \int_{\mathbb{K}} \hat{\nu}^{s}\left(\mathfrak{s}^{-j} \xi\right)\left|\hat{f}\left(\mathfrak{s}^{-j} \xi\right)\right| \hat{\nu}^{s}\left(\mathfrak{s}^{-j}(\xi+\omega(m))\right)\left|\hat{g}\left(\mathfrak{s}^{-j}(\xi+\omega(m))\right)\right|
\end{aligned}
$$




$$
\begin{gathered}
\times\left\{\sum_{r \in D_{1}} \sum_{i \in \mathbb{N}_{0}}\left|\hat{\psi}_{r}^{(j-i)}\left(\mathfrak{s}^{-i} \xi\right)\right|\left|\hat{\psi}_{r}^{(j-i)}\left(\mathfrak{s}^{-i}(\xi+\omega(m))\right)\right|\right\} d \xi \\
\leq \sum_{j \leq J_{0}} \sum_{m \in q \mathbb{N}_{0}+\tilde{Q}} q^{j} \int_{\mathbb{K}} \hat{\nu}^{\frac{s}{2}}\left(\mathfrak{s}^{-j} \xi\right)\left|\hat{f}\left(\mathfrak{s}^{-j} \xi\right)\right| \hat{\nu}^{\frac{s}{2}}\left(\mathfrak{s}^{-j}(\xi+\omega(m))\right)\left|\hat{g}\left(\mathfrak{s}^{-j}(\xi+\omega(m))\right)\right|\left\{\sum_{r \in D_{1}} \sum_{i \in \mathbb{N}_{0}}\right. \\
\left.\hat{\nu}^{s}\left(\mathfrak{s}^{-j} \xi\right)\left|\hat{\psi}_{r}^{(j-i)}\left(\mathfrak{s}^{-i} \xi\right)\right|^{2}+\sum_{r \in D_{1}} \sum_{i \in \mathbb{N}_{0}} \hat{\nu}^{s}\left(\mathfrak{s}^{-j}(\xi+\omega(m))\right)\left|\hat{\psi}_{r}^{(j-i)}\left(\mathfrak{s}^{-i}(\xi+\omega(m))\right)\right|^{2}\right\} d \xi \\
=I_{1}+I_{2},
\end{gathered}
$$

where

$$
\begin{aligned}
I_{1}=\sum_{j \leq J_{0}} \sum_{m \in q \mathbb{N}_{0}+\tilde{Q}} q^{j} \int_{\mathbb{K}} \hat{\nu}^{\frac{s}{2}}\left(\mathfrak{s}^{-j} \xi\right)\left|\hat{f}\left(\mathfrak{s}^{-j} \xi\right)\right| \hat{\nu}^{\frac{s}{2}}\left(\mathfrak{s}^{-j}(\xi+\omega(m))\right)\left|\hat{g}\left(\mathfrak{s}^{-j}(\xi+\omega(m))\right)\right| \\
\times\left\{\sum_{r \in D_{1}} \sum_{i \in \mathbb{N}_{0}} \hat{\nu}^{s}\left(\mathfrak{s}^{-j} \xi\right)\left|\hat{\psi}_{r}^{(j-i)}\left(\mathfrak{s}^{-i} \xi\right)\right|^{2}\right\} d \xi
\end{aligned}
$$

We claim for fixed j, $\int_{\mathbb{K}}\left(\sum_{r \in D_{1}} \sum_{i \in \mathbb{N}_{0}} \hat{\nu}^{s}\left(\mathfrak{s}^{-j} \xi\right)\left|\hat{\psi}_{r}^{(j-i)}\left(\mathfrak{s}^{-i} \xi\right)\right|^{2}\right) d \xi<\infty$.

$$
\begin{aligned}
\int_{\mathbb{K}} \sum_{r \in D_{1}} \sum_{i \in \mathbb{N}_{0}} \hat{\nu}^{s}\left(\mathfrak{s}^{-j} \xi\right)\left|\hat{\psi}_{r}^{(j-i)}\left(\mathfrak{s}^{-i} \xi\right)\right|^{2} d \xi & =\sum_{r \in D_{1}} \sum_{i \in \mathbb{N}_{0}} \int_{\mathbb{K}} \hat{\nu}^{s}\left(\mathfrak{s}^{-j} \xi\right)\left|\hat{\psi}_{r}^{(j-i)}\left(\mathfrak{s}^{-i} \xi\right)\right|^{2} d \xi \\
& =\sum_{r \in D_{1}} \sum_{i \in \mathbb{N}_{0}} \int_{\mathbb{K}} \hat{\nu}^{s}(\xi)\left|\hat{\psi}_{r}^{(j-i)}\left(\mathfrak{s}^{j-i} \xi\right)\right|^{2} q^{-j} d \xi \\
& =q^{-j} \sum_{r \in D_{1}} \sum_{i \in \mathbb{N}_{0}} \int_{\mathbb{K}} \hat{\nu}^{s}(\xi) \frac{q^{i-j}}{q^{i-j}}\left|\hat{\psi}_{r}^{(j-i)}\left(\mathfrak{s}^{j-i} \xi\right)\right|^{2} d \xi \\
& =\sum_{r \in D_{1}} \sum_{i \in \mathbb{N}_{0}} \int_{\mathbb{K}} \hat{\nu}^{s}(\xi) \frac{q^{i-j}}{q^{i}}\left|\hat{\psi}_{r}^{(j-i)}\left(\mathfrak{s}^{j-i} \xi\right)\right|^{2} d \xi \\
& =\sum_{r \in D_{1}} \sum_{i \in \mathbb{N}_{0}} \frac{1}{q^{i}} \int_{\mathbb{K}} \hat{\nu}^{s}(\xi)\left|q^{\frac{(i-j)}{2}} \hat{\psi}_{r}^{(j-i)}\left(\mathfrak{s}^{j-i} \xi\right)\right|^{2} d \xi \\
& =\sum_{r \in D_{1}} \sum_{i \in \mathbb{N}_{0}} \frac{1}{q^{i}}\left\|q^{\frac{(i-j)}{2}} \hat{\psi}_{r}^{(j-i)}\left(\mathfrak{s}^{j-i} \xi\right)\right\|_{H^{s}(\mathbb{K})} \\
& =\sum_{r \in D_{1}} \sum_{i \in \mathbb{N}_{0}} \frac{1}{q^{i}} \times 1<\infty,
\end{aligned}
$$

and

$$
\begin{array}{r}
I_{2}=\sum_{j \leq J_{0}} \sum_{m \in q \mathbb{N}_{0}+\tilde{Q}} q^{j} \int_{\mathbb{K}} \hat{\nu}^{\frac{s}{2}}\left(\mathfrak{s}^{-j} \xi\right)\left|\hat{f}\left(\mathfrak{s}^{-j} \xi\right)\right| \hat{\nu}^{\frac{s}{2}}\left(\mathfrak{s}^{-j}(\xi+\omega(m))\right)\left|\hat{g}\left(\mathfrak{s}^{-j}(\xi+\omega(m))\right)\right| \\
\times\left\{\sum_{r \in D_{1}} \sum_{i \in \mathbb{N}_{0}} \hat{\nu}^{s}\left(\mathfrak{s}^{-j}(\xi+\omega(m))\right)\left|\hat{\psi}_{r}^{(j-i)}\left(\mathfrak{s}^{-i}(\xi+\omega(m))\right)\right|^{2}\right\} d \xi
\end{array}
$$




$$
\begin{aligned}
&=\sum_{j \leq J_{0}} \sum_{m \in q \mathbb{N}_{0}+\tilde{Q}} q^{j} \int_{\mathbb{K}} \hat{\nu}^{\frac{s}{2}}\left(\mathfrak{s}^{-j}(\xi-\omega(m))\right)\left|\hat{f}\left(\mathfrak{s}^{-j}(\xi-\omega(m))\right)\right| \hat{\nu}^{\frac{s}{2}}\left(\mathfrak{s}^{j} \xi\right)\left|\hat{g}\left(\mathfrak{s}^{-j} \xi\right)\right| \\
& \times\left\{\sum_{r \in D_{1}} \sum_{i \in \mathbb{N}_{0}} \hat{\nu}^{s}\left(\mathfrak{s}^{-j} \xi\right)\left|\hat{\psi}_{r}^{(j-i)}\left(\mathfrak{s}^{-i} \xi\right)\right|^{2}\right\} d \xi .
\end{aligned}
$$

Thus $I_{2}$ has same form as $I_{1}$ with the roles of $\hat{f}$ and $\hat{g}$ interchanged. Now we only to show that $I_{1} \rightarrow 0$ as $M \rightarrow \infty$. We can write equation (3.27)

$$
\begin{array}{r}
I_{1}=\sum_{j \leq J_{0}} \sum_{m \in q \mathbb{N}_{0}+\tilde{Q}} q^{j} q^{\frac{M}{2}} \int_{\mathfrak{s}^{-j}} \\
\quad \times\left\{\sum_{r \in D_{1}+\mathfrak{P}^{-j+M}} \sum_{i \in \mathbb{N}_{0}} \hat{\nu}^{s}\left(\mathfrak{s}^{-j} \xi\right)\left|\hat{\psi}_{r}^{(j-i)}\left(\mathfrak{s}^{-i} \xi\right)\right|^{2}\right\} d \xi . \\
\end{array}
$$

Now ,if $\hat{g}\left(\mathfrak{s}^{-j}(\xi+\omega(m))\right) \neq 0$ then we must obtain $\mathfrak{s}^{-j} \xi+\mathfrak{s}^{-j} \omega(m) \in \xi+\mathfrak{P}^{M}+\omega\left(k_{0}\right)$ and $\left|\mathfrak{s}^{-j} \omega(m)\right| \leq q^{-M}$, and hence $|\omega(m)| \leq q^{-M-j}$. Then the summation index is bounded by $q^{-M-j}$, so using this we get

$$
\begin{aligned}
I_{1} & \leq \sum_{j \leq J_{0}} q^{j} q^{\frac{M}{2}} \int_{\mathfrak{s}^{-j} \xi_{0}+\mathfrak{P}^{-j+M}} \sum_{r \in D_{1}} \sum_{i \in \mathbb{N}_{0}} \hat{\nu}^{s}\left(\mathfrak{s}^{-j} \xi\right)\left|\hat{\psi}_{r}^{(j-i)}\left(\mathfrak{s}^{-i} \xi\right)\right|^{2} q^{\frac{M}{2}} q^{-M-j} d \xi \\
& \leq \sum_{j \leq J_{0}} \int_{\mathfrak{s}^{-j} \xi_{0}+\mathfrak{P}^{-j+M}} \sum_{r \in D_{1}} \sum_{i \in \mathbb{N}_{0}} \hat{\nu}^{s}\left(\mathfrak{s}^{-j} \xi\right)\left|\hat{\psi}_{r}^{(j-i)}\left(\mathfrak{s}^{-i} \xi\right)\right|^{2} d \xi .
\end{aligned}
$$

For given $\xi_{0}$, for any $\epsilon>0$, choose $k<0$ enough small satisfies the following two inequalities $q^{k}<|\xi|=q^{\rho}$ such that $k+\rho<0$, and

$$
\int_{\mathfrak{P}^{-k-\rho}} \hat{\nu}^{s}\left(\mathfrak{s}^{-k} \xi\right)\left|\hat{\psi}_{r}^{(k-i)}\left(\mathfrak{s}^{-i} \xi\right)\right|^{2} d \xi<\epsilon .
$$

We have $\mathfrak{s}^{-j} \xi_{0}+\mathfrak{P}^{-j+M} \subset \mathfrak{P}^{-k-\rho}$ for all $j \leq k$. Since $\left|\mathfrak{s}^{-j} \xi_{0}\right| \leq q^{k} q^{q}$ and $\mathfrak{P}^{-j+M} \subseteq \mathfrak{P}^{-k-\rho}$ Hence $I_{1} \rightarrow 0$ as $M \rightarrow \infty$.

Example 1. Assume that the function $\psi_{r}^{(j)}=\frac{q^{j}}{q-1}\left\{\nu^{\frac{-s}{2}}\left(\mathfrak{s}^{-j} x\right)\right\} *\left(\chi_{\mathfrak{D}}(x)-\phi(x)\right)$ for all $j \in \mathbb{Z}, r=1,2,3,4, \ldots .(q-1)$, where $\chi_{\mathfrak{D}}$ is the characteristic function on $\mathfrak{D}$ and $\phi(x)=\left\{\begin{array}{cl}q^{-1}, & \text { if } x \in \mathfrak{P}^{-1}, \\ 0, & \text { otherwise }\end{array}\right.$ Thus, $\quad \hat{\psi}_{r}^{(j)}(\xi)=\left\{\begin{aligned} \frac{1}{q-1} \hat{\nu}^{\frac{-s}{2}}\left(\mathfrak{s}^{j} \xi\right), & \text { if } \xi \in \mathfrak{P}^{-1} \backslash \mathfrak{D}, \\ 0, & \text { otherwise }\end{aligned}\right.$ For $\xi \neq 0$, we note that

$$
\sum_{r=1}^{q-1} \sum_{j \in \mathbb{Z}}\left|\hat{\psi}_{r}^{(j)}\left(\mathfrak{s}^{-j} \xi\right)\right|^{2}=\frac{1}{q-1} \sum_{r=1}^{q-1} \hat{\nu}^{-s}(\xi)=\hat{\nu}^{-s}(\xi) .
$$


Now, it is to see that both $\mathfrak{s}^{-j} \xi$ and $\mathfrak{s}^{-j}\left(\xi+w\left(s^{\prime}\right)\right)$ are not lies in $\mathfrak{P}^{-1} \backslash \mathfrak{D}$ simultaneously. Therefore, equation (3.6) holds. Hence the system $\left\{\psi_{r}^{(j)}: j \in \mathbb{Z}, r=1,2,3, \ldots q-1\right\}$ forms orthonormal wavelet of $H^{s}(\mathbb{K})$.

\section{Acknowledgement}

The authors are thankful to the referee for his/her through review and highly appreciate the comments and suggestions to various improvements in the original manuscript. The research of the first author is supported by Statrup grant to new faculties under XII plan grant No. 5006 (BHU), UGC, New Delhi, India and second author is supported by University Grants Commission ( UGC), grant number: F.No. 16-6(DEC. 2017)/2018(NET/CSIR), New Delhi, India.

\section{References}

[1] A. Pathak, Continuous Wavelet Transform on Local Fields, Bol. Soc. Paran. Mat. 34 (2016), 113-121.

[2] A. Pathak and D. Kumar, Characterization of Multiwavelets and MRA Wavelets in $H^{s}(\mathbb{F})$, International Journal of Applied and Computational Mathematics 5(6) (2019), 143.

[3] A. Pathak and D. Kumar, Existence of unconditional wavelet basis of $L^{p}$ - norm over a local fields of positive characteristic (communicated).

[4] A. Pathak, D. Kumar and G. P. Singh, The Necessary Condition and Sufficient conditions for Wavelet Frames in Sobolev Space over Local field of Positive characteristic, Bol. Soc. Paran. Mat., 39(3)(2021) 81-92.

[5] A. Pathak and G. P Singh, Wavelet in Sobolve space over local fields of positive characteriscic, Int. J. of Wavelets Multiresolunt. Inf. Process 3 (2018), 16.

[6] A. Pathak and G. P. Singh, Biorthogonal Wavelets in Sobolev Space Over Local Fields of Positive Characteristic. International Journal of Applied and Computational Mathematics 6(2) (2020), 25.

[7] A. Pathak and G. P. Singh, Multilevel Wavelet packets in Sobolev space over local fields of positive characteristic (communicated).

[8] A. Ron and Z. Shen, Frames and stable bases for shift invariant subspaces of $L^{2}\left(\mathbb{R}^{d}\right)$. Can. J. Math. 47(1995), 1051-1094. 
[9] A. Ron and Z. Shen, Affine systems in $L^{2}\left(\mathbb{R}^{d}\right)$ the analysis of the analysis operator. J. Funct. Anal. 148 (1997), 408-447.

[10] B. Behera, Haar wavelets on the Lebesgue spaces of local fields of positive characteristic, Colloquium Mathematicum. 2 (2014), 149-168.

[11] B. Behera and Q. Jahan, Multiresolution analysis on local fields and characterization of scaling functions, Adv. Pure Appl. Math. 3 (2012), 181-202.

[12] B. Behera and Q. Jahan, Characterization of wavelets and MRA wavelets on local fields of positive characteristic, Collect. Math. 66 (2015), 33-53.

[13] C. K. Chui, An Introduction to Wavelets, Wavelet Analysis and Its Applications 1, Academic Press, Boston, MA, (1992).

[14] C. Chui, X. Shi and J. Stocker, Affine frames, quasi-affine frames, and their duals. Adv. Comput. Math. 8 (1998), 1-17.

[15] D. F. Walnut, An introduction to wavelet analysis, Birkhauser (2002).

[16] E. Hernández and G. Weiss, A First Course on Wavelets, CRC Press, Boca Raton,FL, (1996).

[17] G. Gripenberg, A necessary and sufficient condition for the existance of a father wavelet. Stud. Math.114 (1995), 207-226.

[18] H. Jiang, D. Li and N. Jin, Multiresolution analysis on local fields, J. Math. Anal. Appl. 294 (2004), 523-532.

[19] I. Daubechies, Ten Lectures on Wavelets, CBMS-NSF Regional Conference Series in Applied Mathematics, Society for Industrial and Applied Mathematics (SIAM), Philadelphia, PA, 61 (1992).

[20] J. J. Benedetto and R. L. Benedetto, A wavelet theory for local fields and related groups, J. Geom. Anal. 14 (2004), 423-456.

[21] M. Bownik, On characterization of multiwavelets in $L^{2}\left(\mathbb{R}^{n}\right)$.Proc. Am. Math. Soc. 129 (2001), 3265-3274.

[22] M. Bownik, The structure of shift invariant subspaces of $L^{2}\left(\mathbb{R}^{n}\right)$. J. Funct. Anal. 177 (2000), 282-309.

[23] M. H. Taibleson, Fourier Analysis on Local Fields, Mathematical Notes, Princeton University Press, Princeton, NJ 15 (1975). 
[24] R. L. Benedetto, Examples of wavelets for local fields, in: Wavelets, Frames and Operator Theory, Contemporary Mathematics, American Mathematical Society, Providence, RI 345 (2004), 27-47.

[25] S. Dahlke, Multiresolution analysis and wavelets on locally compact abelian groups. in: Wavelets, images, and surface fitting, A K Peters (1994), 141-156pp.

[26] S. Mallat, Multiresolution approximations and wavelet orthonormal bases of $L^{2}(\mathbb{R})$, Trans. Amer. Math. Soc. 315 (1989), 69-87.

[27] X. Wang, The study of wavelets from the properties of their Fourier transform, PhD. Thesis, Washington University (1995).

Ashish Pathak Department of Mathematics, Institute of Science, Banaras Hindu University, Varanasi-221005, India.

E-mail: ashishpathak@bhu.ac.in

Dileep Kumar Department of Mathematics, Institute of Science, Banaras Hindu University, Varanasi-221005, India.

E-mail: dkbhu07@gmail.com 